\title{
X-ray emission from A-type stars ${ }^{\star}$
}

\author{
C. Schröder and J. H. M. M. Schmitt
}

\author{
Hamburger Sternwarte, Universität Hamburg, Gojenbergsweg 112, 21029 Hamburg, Germany \\ e-mail: cschroeder@hs.uni-hamburg.de
}

Received 7 March 2007 / Accepted 10 September 2007

ABSTRACT

\begin{abstract}
Being fully radiative, stars of spectral type A are not expected to harbor magnetic dynamos and hence such stars are not expected to produce X-ray emission. Indeed, while the X-ray detection rate of such stars in X-ray surveys is low, it is not zero and some of the brighter A-type stars have been detected on different occasions and with different instruments. To study systematically the puzzle of the X-ray emitting A-type stars, we carried out an X-ray study of all A-type stars listed in the Bright Star Catalogue using the ROSAT public data archive. We found a total of 312 bright A-type stars positionally associated with ROSAT X-ray sources; we analyzed the $\mathrm{X}$-ray light curves as well as searched for evidence of RV variations to identify possible late-type companions producing the X-ray emission. In this paper we present a list of X-ray active A-type stars, including the collected data about multiplicity, X-ray luminosity and spectral peculiarities.
\end{abstract}

Key words. X-rays: stars - stars: activity - stars: coronae - stars: general

\section{Introduction}

Many if not all late-type, main-sequence stars are surrounded by X-ray emitting coronae. Extensive ROSAT surveys (Schmitt \& Liefke 2004) show that the X-ray detection rates among the brighter late-type stars with spectral type $\mathrm{F}$ and later are approaching $100 \%$; the same holds for early-type stars surveyed by Berghöfer et al. $(1996,1997)$, although the X-ray production mechanisms in the early-type stars is thought to be fundamentally different from that in late-type stars. The X-ray production mechanism for the late-type stars is believed to be similar to that of the Sun, and indeed, the dramatic increase in X-ray detection rates for stars thought to possess surface convection zones lends support to the idea that the stellar magnetism, which is ultimately held responsible for the observed activity phenomena, is generated in the turbulent outer layers of late-type stars. Early-type stars on the other hand produce strong radiatively driven winds, and instabilities in these winds are thought to be ultimately responsible for the ubiquitous X-ray emission observed from these stars. A general paucity of X-ray emission among (late-type) Band especially A-type stars has been noticed ever since the days of the Einstein Observatory (Schmitt et al. 1985). Since A-type stars have neither convection zones nor do they have - at least strong winds, none of the X-ray production mechanisms operating in early- or late-type stars ought to be operating and therefore A-type stars are "expected" to be intrinsically X-ray dark.

This expectation of intrinsic X-ray darkness/weakness is borne out by the available X-ray data on nearby A-type stars. The nearest A-type stars are Sirius and Vega, but the signals seen in the ROSAT X-ray detectors (especially the ROSAT-HRI) are in all likelihood produced by UV-radiation rather than genuine X-ray radiation (Zombeck et al. 1997; Berghöfer et al. 1999). A further, and more severe problem for the interpretation of X-ray data from A-type stars is binarity. Because of the ubiquity of

* Tables 2 et 3 are only available in electronic form at http://www . aanda.org
X-ray emission among late-type stars, all A-type stars with a late-type companion should be X-ray sources, which are simply attributable to the presence of late-type companions. Since such optically, possibly quite faint, companions are easy to hide in the vicinity of an optically bright A-type star, the X-ray emission from even presumably single A-type stars could in fact attributed to previously unknown late-type companions. Because of the rather short main sequence life times of A-type stars latetype companions of such stars are still considered young, hence most of the "hidden" late-type companions are expected to be $\mathrm{X}$-ray bright and active sources.

One of the unique attributions of the observed X-ray emission in a stellar system is quite difficult; it requires X-ray data with sufficient angular resolution, which would actually still be insufficient to rule out close companions. Stelzer et al. (2006) used Chandra imaging observations to spatially resolve a sample of main sequence B-type stars with recently discovered companions at arcsecond separation. They found that all spatially resolved companions are X-ray emitters, but - somewhat surprisingly - seven out of eleven B-type stars were also found to be X-ray sources. Obviously this result still does not rule out additional close companions of the B stars in the system, but would imply a rather large binary frequency of intermediate-mass stars and a high number of systems with more than two components.

Another technique is observing systems with a special viewing geometry such as an eclipsing binary. Schmitt \& Kürster (1993) and Schmitt (1998) studied the (totally) eclipsing binary $\alpha \mathrm{CrB}$, consisting of an A0V primary and a G2V secondary. At secondary optical minimum with the optically bright A-type star in front of the optically fainter G-type star a total X-ray eclipse was observed, thus demonstrating, first, that the A-type primary is indeed X-ray dark "as expected", and second, that the size of the X-ray corona around the secondary is at least smaller than the size of the primary.

In a few other cases, the correct attribution of observed $\mathrm{X}$-ray emission from A-type stars can be made using the spectral 
information contained in the X-ray data. In the prototypical case $\beta$ Crt (Fleming et al. 1991) the soft PSPC spectrum suggested the presence of an X-ray emitting white dwarf, which was subsequently confirmed (Barstow et al. 1994; Vennes et al. 1998). Thus $\beta$ Crt is very similar to the binary system Sirius AB with its prototypical white dwarf; if this system was ten times further away from the Sun, it would still be quite X-ray bright, but the B component would be almost impossible to detect at least at optical wavelengths.

Obviously the set of - for whatever reasons - X-ray bright A-type stars is a bewildering zoo of quite different beasts. The purpose of this paper is to provide a definitive summary of the ROSAT observations of bright A-type stars. If the puzzle of $\mathrm{X}$-ray emitting A-type stars is ever to be solved, a well defined sample of the brightest of such objects is required. The X-ray emitting A-type stars were detected and identified and an extensive literature search was carried out to single out those objects whose X-ray emission cannot be explained by emission from late-type companions, at least with the currently available information on these objects.

\section{Observations and data analysis}

\subsection{Data basis}

The ROSAT Observatory was operated between 1990-1998. Between July 1990 and January 1991 it carried out its ROSAT All-Sky Survey (RASS) with the ROSAT Position Sensitive Proportional Counter (PSPC). Afterwards pointed observations of individual objects were carried out within the framework of the ROSAT guest investigator program both with the PSPC and a High (angular) Resolution Imager (HRI). These detectors had fields of view of about $7000 \mathrm{arcmin}^{2}$ for the PSPC and $1000 \operatorname{arcmin}^{2}$ for the HRI, so that many X-ray sources were picked up serendipitously in the field of view of many observations, whose original scientific goal was actually quite different.

\subsubsection{X-ray data}

The results of both the RASS observations and the ROSAT pointed observations are available in the ROSAT results archive in the form of source lists. Four of the five catalogs were used for the present study, i.e., the ROSAT Bright Source and Faint Source Catalog, the Second ROSAT Source Catalog of Pointed Observations with the PSPC and the First ROSAT Source Catalog of Pointed Observations with the HRI. These catalogs as well as detailed information on the detection and screening procedures applied in the construction of the catalogs are available via www from the ROSAT Home Page at Max-Planck-Institut für extraterrestrische Physik (http://wave.xray.mpe.mpg.de/rosat/catalogue) or its mirror sites. Altogether, these catalogs contain 372896 entries. From the data provided by these catalogs we extracted position and count rates of those sources, which can be positionally associated with bright A-type stars. For those sources observed with the PSPC with the RASS, we used the X-ray luminosities derived by Hünsch et al. (1998).

\subsubsection{Optical data}

As our source of optical data we used the 5th version of the Bright Star Catalog (BSC) by Hoffleit \& Jaschek (1991), which contains 9110 objects of magnitude 6.5 or brighter. For the parallax values we used the data given in the Hipparcos database.
Out of those 9110 objects, 1966 stars are listed as A-type stars in the BSC. The BSC thus constitutes a very large and complete database of bright A-type stars with a lot of auxiliary information about its entries. For information concerning possible hidden late-type companions of the A-type stars we used the SIMBAD database extensively.

\subsubsection{Catalogs}

The catalogs in Tables 2 and 3 present the main result of our efforts; in Table 2 we provide optical and X-ray data of 84 detected "bona fide" single A-type stars as contained in the BSC. Table 3 shows the same data for 228 X-ray associated A-type stars that are members of known binary or multiple systems or show signs of hidden companions. The columns of the table contain the following information:

Col. 1: HR number, taken from the BSC;

Col. 2: binary flag; S means single star, B visual or eclipsing binary, SB spectroscopic binary, PB potential binary;

Col. 3: rotational velocity in $\mathrm{km} \mathrm{s}^{-1}$;

Col. 4: separation for visual binary systems;

Col. 5: difference in the magnitude between the A star and its closest companion;

Col. 6: MK spectral types of the components;

Col. 7: distance of the star as given by Hipparcos parallaxes;

Col. 8: catalog flag indicating in which observation mode the star has been observed; r means ROSAT all-sky survey, p PSPC pointing and $\mathrm{h}$ HRI pointing;

Col. 9: mean count rate of the associated X-ray source; first choice were PSPC pointing source data, second HRI and third survey observation data;

Col. 10: error of the count rate; errors with a value of 0.0 mark sources with flags in the ROSAT catalogs that indicate a suspect source or false detection in the field;

Col. 11: X-ray luminosity derived from the distance as given in Col. 7 and the count rates as given in Col. 9. The values are given in units of $10^{27} \mathrm{erg} / \mathrm{s}$.

\subsection{Data analysis}

\subsubsection{Correlation of the catalogs}

For the correlation between the X-ray and the optical source lists we combined the ROSAT Faint Source Catalog and the ROSAT Bright Source Catalog to the RASS catalog. The resulting three X-ray catalogs and the Bright Star Catalog were searched for positional coincidences. As a matching criterion we used 90 arcsec for survey data, 36 arcsec for pointing data with the PSPC and 18 arcsec for HRI data. The differently chosen positional acceptance thresholds reflect the fact that the intrinsic positional accuracy of survey data, and the PSPC and HRI pointing data increases in that order. In Fig. 1 we plot histograms showing the number of sources obtained by our positional correlation analysis as a function of angular distance. The concentration of the sources towards the target positions, i.e., the 1966 A-type stars in the BSC catalog, is obvious. As shown in Fig. 1 the matching criteria approximately mark the distances at which the probability of obtaining a spurious X-ray source becomes greater than the chance to obtain a correct identification. Therefore the chosen values for the acceptance thresholds do not introduce significant errors into our list of seemingly X-ray active A-type stars. 


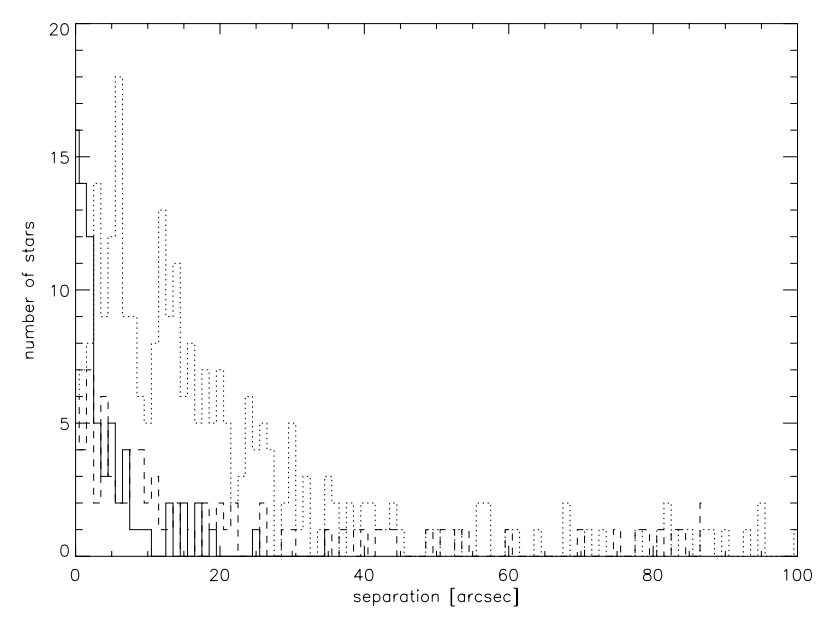

Fig. 1. Number of A-type stars at a given separation between the optical position of the star and the position of the associated X-ray source. The dotted line shows the data for the BSC-RASS correlation, the dashed line represents the BSC-PSPC data and the solid line those from the BSC-HRI correlation.

\subsubsection{Search for late-type companions}

The most common explanation for seemingly X-ray active A-type stars is to assume that the $\mathrm{X}$-ray emission does not originate from the A-type star itself but rather from a low mass (binary) companion. To check this hypothesis we searched for signatures of hidden companions in four ways. First, we examined possible variations in the radial velocity and proper motion of the A-type star given in the SIMBAD database and accordant papers. Second, we checked the ROSAT X-ray light curves for flare-like variations which might be an indicator of the presence of a late-type companion. Third, for those stars observed in more than one observation mode we performed a comparison of the count rates in the different observations to check for long time scale variability. Different count rates at the two different observation times are an indicator for a variable source and therefore hint at the presence of a hidden companion. Admittedly, the significance of this indicator is small, since the possible mechanisms for the X-ray emission from A-type star are somewhat speculative. Finally, another, albeit only weak, indicator is the rotational velocity of the A-type star itself. Given enough time to synchronize their orbits, the members of a binary system would rotate slowly and a high rotational velocity might therefore indicate a single star or a young binary system. The results of these tests are included in our list of seemingly X-ray active A-type stars. Stars without any available information on binarity and no signatures of hidden companions we consider as bona fide single stars.

\section{Results}

On basis of the correlation of the Bright Star Catalogue and the three ROSAT catalogs we can associate 312 of the 1966 A-type stars with one or more X-ray sources. In total, 272 of these sources are listed as RASS detected X-ray sources, 63 sources are listed in the PSPC pointing catalog and 69 have been observed at the highest possible angular resolution with the ROSAT HRI. The total number of these sources is larger than 312 because some stars have been detected in more than one mode/instrument.
Table 1. Statistics of spectral peculiar A-stars.

\begin{tabular}{ccccc}
\hline \hline & Total & $\begin{array}{c}\text { X-ray } \\
\text { detected }\end{array}$ & $\begin{array}{c}\text { Known } \\
\text { binaries }\end{array}$ & $\begin{array}{c}\text { Binaries among } \\
\text { X-ray detections }\end{array}$ \\
\hline A-type stars & 1966 & $312(16 \%)$ & $733(37 \%)$ & $194(62 \%)$ \\
Am & 212 & $41(19 \%)$ & $89(42 \%)$ & $34(83 \%)$ \\
Ap & 133 & $18(14 \%)$ & $51(38 \%)$ & $8(44 \%)$ \\
A giants & 474 & $62(13 \%)$ & $179(38 \%)$ & $35(56 \%)$ \\
\hline
\end{tabular}

\subsection{Spurious identifications}

We stress that all our X-ray source identifications are obtained solely on the basis of positional coincidence. Despite of this we expect the number of spurious identifications to be very small. Calculating the number of identifications obtained by distributing approximately 120000 RASS sources over 1966 positions (i.e., the number of A-type stars contained in the BSC) with a detected cell radius of 90 arcsec results in 11 spurious identifications or $4 \%$ of the total number of RASS detections of A-type stars. The actual distribution of position offsets is much narrower (cf., Fig. 1). To be specific, only 4 out of our 272 survey detections have position offsets of more than $50 \mathrm{arcsec}$, and only $11 \mathrm{X}$-ray detections are off by more than 30 arcsec from the optical positions of the A-type star. We thus conclude that the fraction of incorrectly identified X-ray emitters in our sample is at the one to two percent level and that the overwhelming majority of our sources are correctly identified with an optically bright A-type star.

\subsection{Detection statistics}

Next we divide the 1966 A-type stars as listed in the BSC into four subgroups according to their spectral features: Am, Ap, giant and main sequence stars. To check whether the X-ray activity of A-type stars depends on this grouping we determined the fraction of X-ray active stars in all of the different subgroups. In Table 1 we list the total number of A-type stars, the number of seemingly X-ray active stars, the number of known binary or multiple systems and the binary frequency among the X-ray active stars.

Given the count statistics there are obviously no significant differences in the fraction of X-ray active stars between the different subgroups. However, the X-ray detection rates among the known binaries are larger than the binary fraction in the corresponding subsamples. We interpret this strong increase of the binary frequency in the X-ray active subsamples as an indicator for a larger number of binary systems. These systems, which can be optically separated in quite a number of cases, cannot be separated in the ROSAT X-ray data and therefore cause possibly false correlations between the A-type star and the X-ray source (i.e. the companion). Clearly, the number of known binaries among all A-type stars has to be considered a lower limit. While the sample of seemingly X-ray active stars was intensively searched for signatures of binarity, the remaining 1654 A-type stars, which have not been detected in $\mathrm{X}$-rays, have been classified only according to the multiplicity information given in the BSC.

\subsection{Detection rate vs. spectral type}

In Fig. 2 we plot the detection rate of A-type stars vs. spectral type. As is obvious from Fig. 2, the mean percentage of X-ray 


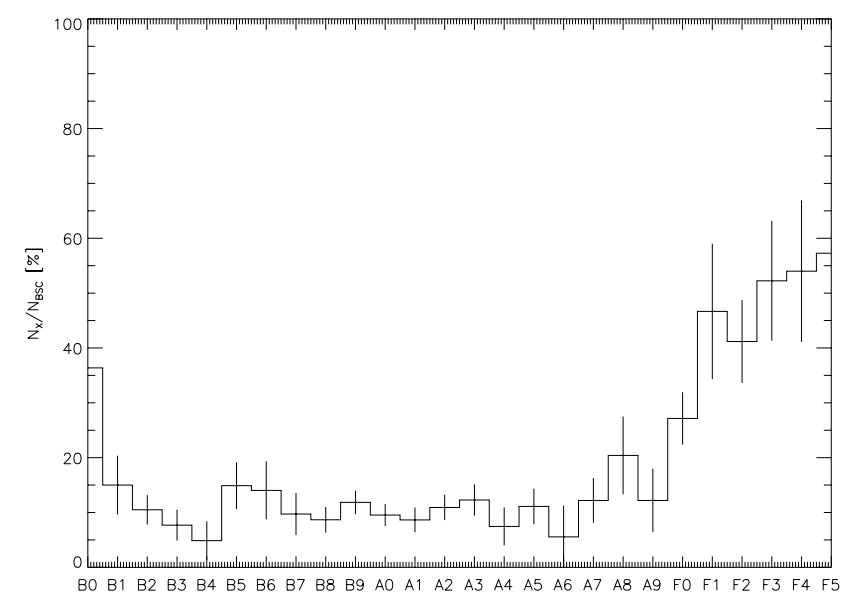

Fig. 2. Percentage of X-ray active stars $N_{\mathrm{x}}$ from the total number of stars $N_{\text {BSC }}$ of a given spectral type. Note the strong increase in the X-ray detection rates for stars in the range A9-F3.

active stars among the stars with spectral type A0 to A9 is $10 \%$ to $15 \%$. This is consistent with the X-ray activity frequency in the above mentioned special subgroups in Table 1 . The onset of convection and hence the presumed onset of an $\alpha \Omega-$ dynamo at spectral type A9-F3 and the radiation driven winds at early B-type stars are clearly visible. The fact that less than $60 \%$ of the F5 stars are X-ray active in our data we attribute to the flux limit of the X-ray data; if instead, one considers a volume-limited sample of F-type stars (Schmitt \& Liefke 2004), one finds a detection rate of essentially $100 \%$.

\subsection{X-ray luminosity}

Figure 3 shows the X-ray luminosity of the ROSAT sources associated with the $312 \mathrm{X}$-ray detected A-type stars as a function of stellar distance; the parallaxes for the stars have been taken from the Hipparcos catalog (ESA 1997). The two objects with the faintest X-ray sources are the AV5 star $\beta$ Pictoris (HD 39060) and the AV7 star $\delta$ Doradus (HD 39014). In the case of $\beta$ Pictoris the recorded ROSAT HRI signal is completely consistent with being due to UV contamination (Hempel et al. 2005), in the case of $\delta$ Doradus this is extremely likely. Berghöfer et al. (1999) derived a relation between the $U, B$ and $V$ magnitudes and the HRI count rates. The predicted count rate for $\delta$ Doradus of $0.24 \mathrm{cts} / \mathrm{ks}$ corresponds to the observed HRI count rate of $0.63 \mathrm{cts} / \mathrm{ks}$ within the errors. The dotted line represents the typical detection limit for the RASS observations, i.e., $f_{\mathrm{X}} \approx 10^{-13} \mathrm{erg} \mathrm{cm}^{-2} \mathrm{~s}^{-1}$. Sources with a lower $L_{\mathrm{X}}$ have been observed with the PSPC or HRI via pointing mode, resulting in deeper observations with lower detection limits. Figure 3 suggests the single and double stars from our X-ray detected sample do not possess significantly different X-ray luminosities as a function of distance.

The distribution functions of the X-ray luminosities of single and binary stars are shown in Fig. 4. Carrying out a formal test with a one-sided Kolmogorov-Smirnov test allows us to reject the null hypothesis that in both classes the X-ray originate from the same population, with a significance of $95 \%$, according to a maximum difference of 0.158 between the two distributions. Additional bootstrap tests with two artificial samples of randomly chosen X-ray detected stars and the same size as the original samples do not support this high level of significance. Out of 10000 runs 2127 show distributions in which the

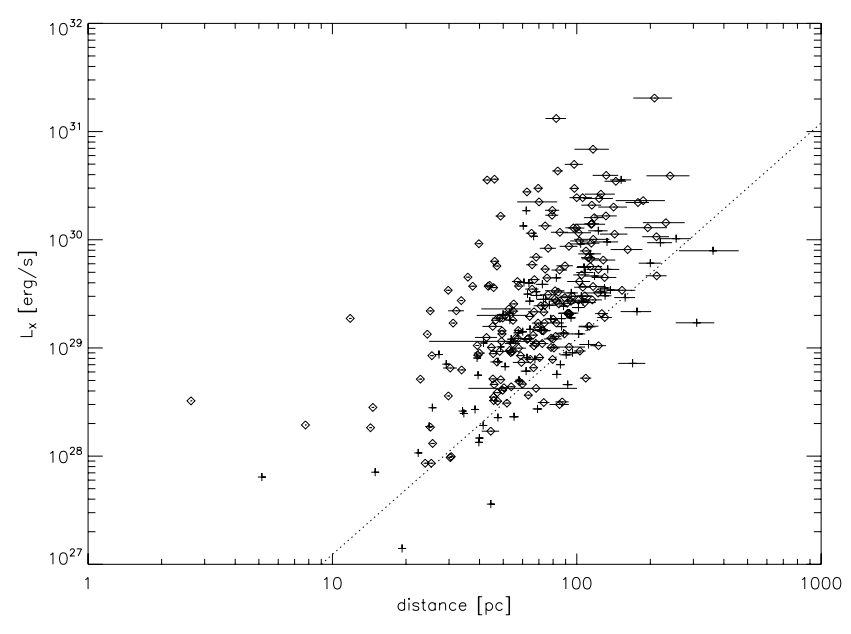

Fig. 3. $L_{x}$ vs. distance for the 312 A-type stars associated with a ROSAT X-ray source. The crosses indicate single stars, and the diamonds known or possible multiple systems. The dotted line represents the typical detection limit for the RASS observations, i.e., $f_{\mathrm{X}} \approx$ $10^{-13} \mathrm{erg} \mathrm{cm}^{-2} \mathrm{~s}^{-1}$.

maximum difference between the distributions is larger than the measured value of 0.158 , hence the bootstrap test suggests only an $80 \%$ confidence for the rejection of the hypothesis that the two samples originate from the same population. We note that the offset in the cumulative distribution function might be a result of a observational bias. The problem is that binary systems with an A-type star and a low mass companion are harder to resolve than systems with a higher mass companion and therefore more likely to be misidentified as a single star. For solar-like companions this would create a "single star" sample with lower X-ray luminosity, since $L_{\mathrm{X}}$ scales with $L_{\mathrm{bol}}$. This effect might be negated by the fact that for M-type stars, especially young ones which are to be expected in binary systems with A-type stars, the difference in X-ray luminosity vanishes (Stauffer et al. 1994; Stern et al. 1995; Randich et al. 1996).

\subsection{Completeness}

Since the absolute magnitudes of our sample stars vary quite a lot, our sample is definitely not volume-limited. Considering a distance limit of 50 parsecs our sample is definitely complete in the sense that all A-type stars up to that distance are sample members. Furthermore, since from the typical RASS flux limit we can compute the RASS upper X-ray luminosity limit of $u l=2.4 \times 10^{25} \times d_{\mathrm{pc}} 2 \mathrm{erg} / \mathrm{s}$, which translates into an X-ray luminosity of $L_{\mathrm{X}}=6 \times 10^{28} \mathrm{erg} / \mathrm{s}$, and thus the X-ray luminosity distribution faction above that limit is unaffected by any nondetections. In the $50 \mathrm{pc}$ volume there are 220 cataloged A-type stars, out of which 82 (or 37\%) are associated with an X-ray source; in other words, the observed detection rate in a volumelimited sample of A-type stars significantly exceeds $15 \%$. The upper limits to the X-ray luminosity of most of the 138 nondetections are in the range $\approx 10^{28} \mathrm{erg} / \mathrm{s}$ for the RASS data; a few nearby A-type stars with upper limits from dedicated pointings (Schmitt 1997; Pease et al. 2006) have upper limits considerably below this value; these stars include $\alpha$ Lyr (A0V), $\beta$ Leo (A3V) and $\alpha$ PsA (A3V). Out of these $82 \mathrm{X}$-ray detcted stars, 60 objects (or $73 \%$ of the X-ray detected stars) are known or supposed to have one or more companions, while 22 stars (or 27\%) of the X-ray detected stars are "bona fide" single stars. The cumulative 


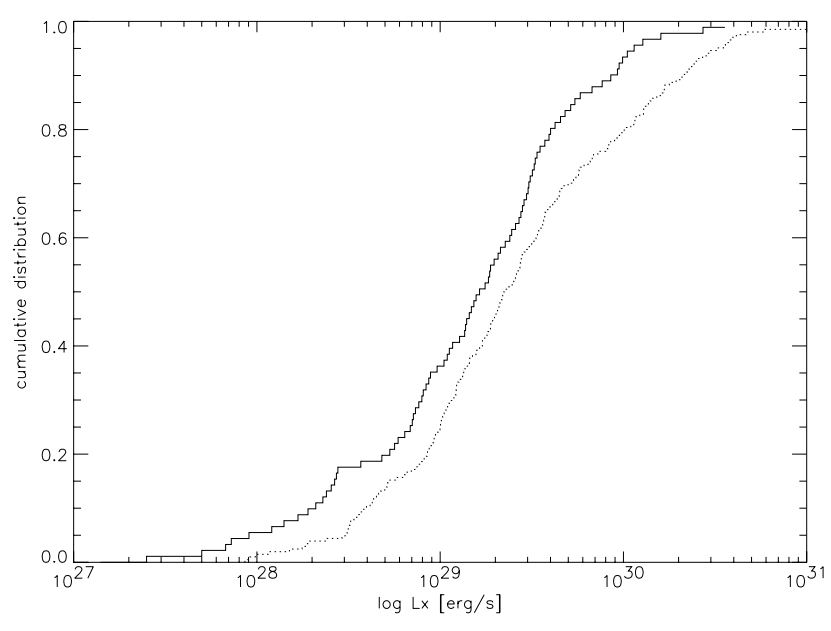

Fig. 4. Cumulative distribution function of the X-ray luminosity for the single star sample (solid line) and binary star sample (dotted line). The null hypothesis that both samples originate from the same distribution can be rejected with a significance of $95 \%$.

distribution functions of the 22 single stars and 60 known binary stars shows a comparable offset as the samples presented in Sect. 3.5, they do of course, suffer from the same possible bias(es). Thus we conclude that a volume-limited sample yields similar properties for X-ray detected A-type stars, except that the true detection rate maybe quite a bit higher.

\subsection{Variability between survey and pointing data}

For some of our sample stars multiple X-ray detections are available from RASS data, the ROSAT PSPC pointing or HRI pointing program. The time span between survey and pointed observations with the PSPC is typically of the order of 1-2 years with larger time spans between the survey and the pointed observations carried out with the HRI instrument.

In Fig. 5 we plot the PSPC count rate observed during the allsky survey vs. the PSPC count rate observed during the pointing program in a double-logarithmic representation for those of our sample stars detected in both observing modes. The two dotted lines indicate a factor of two deviation above and below unity. As Fig. 5 shows, all count rates lie within this area according to their error bars. Variations by a factor of 4 are not uncommon for active late-type stars and would therefore support the late-type companion hypothesis, as does the fact that there is no significant difference in the variability of single and binary stars.

The variability between the count rates obtained during the PSPC pointed observations and the HRI pointed observations is shown in Fig. 6. Again, the dotted lines mark a factor of two deviation above and below "unity"; "unity" in this case accounts for the fact that the ROSAT-HRI instrument is less sensitive than the ROSAT-PSPC leading to an HRI count rate typically a quarter of the PSPC count rate. Only HR 5999 shows a variation in count rate differing by a factor of 12 ; however, we suspect that the X-ray emission originates from the possible T Tauri star Rossiter 3930, which is separated to HR 5999 by 1.3" (Zinnecker \& Preibisch 1994; Stecklum et al. 1995), also, HR 5999 lies in a crowded field not resolved in the survey data.

Figure 7 shows the third possible combination of the three catalog data sets, the comparison of the count rates obtained during the PSPC survey program and the HRI pointing program. As in Fig. 6, the dotted line indicates "unity" which takes into

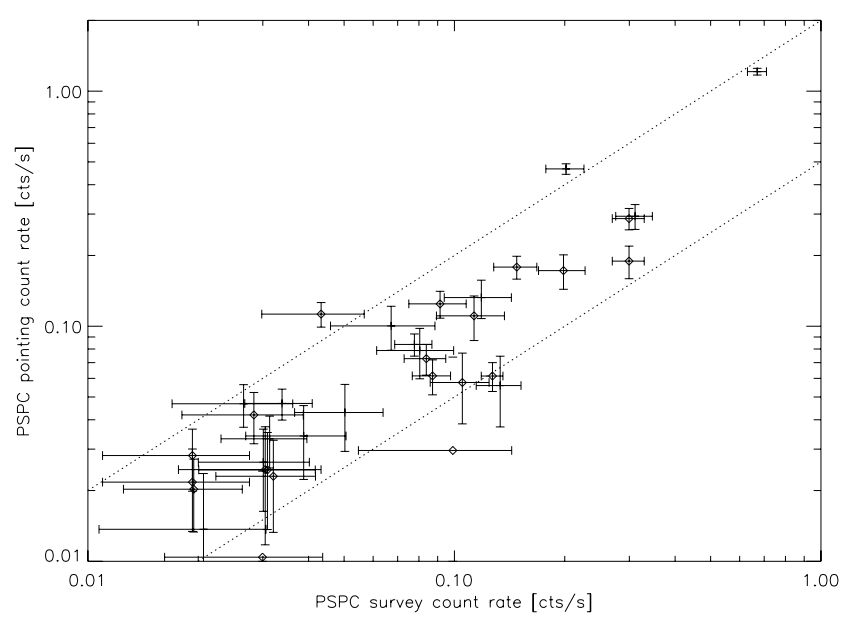

Fig. 5. Comparison of X-ray count rates for 35 stars detected both in the all-sky survey and the PSPC pointing program. The two dotted lines indicate a factor 2 variation from unity. Single and double stars are represented by crosses and diamonds respectively. Stars with large deviations from the regression line are identified.

account the lower sensitivity of the ROSAT-HRI instrument. Five stars show apparent count rate variations. HR 1189 is a binary system with a separation of $8^{\prime \prime}$, containing an A1V and a late B-type star. Due to the fact that the survey observation has an exposure time of only $70 \mathrm{~s}$, it is most likely that the variation originates from the uncertainty in the count rate of the RASS source. HR 2890 (Castor B) is a spectroscopic binary with spectral types of A5Vm and early M (Gudel \& Schmitt 1996). Closeby, with a separation of $3.9^{\prime \prime}$, lies the spectroscopic binary system Castor A, which is composed of an A1V and a late K-star. Due to the configuration of these two binary systems the X-ray emission and its variability can be clearly associated with the late-type companions of the A-type stars (Güdel et al. 2001). HR 3524 (RS Cha) is an eclipsing binary consisting of two A8V stars (Andersen 1975). In contrast to earlier publications, Mamajek et al. (2000) derived ages of $5.0 \times 106$ and $4.3 \times 106 \mathrm{yr}$ for the two components and suggest that this system, because of the fact that the X-ray emission of RS Cha shows flare-like variations, is actually a triple system with an undetected T Tauri star. According to the Washington Visual Double Star Catalog, HR 8662 is a visual binary with a separation of 10.5" and additionally the A component is a spectroscopic binary. Corbally (1994) specifies the spectral type of the B component as G9V, thus giving a likely explanation for the count rate variations, keeping in mind the resolving power during the survey observations.

\subsection{Individual objects of pointed HRI observations}

In the following we present some ROSAT HRI observations of X-ray detected A-type stars for which PSPC and high resolution ROSAT HRI observations are available. Additionally, detailed information about these stars has been published in different papers and might give hints about the absence or presence of undetected late-type companions.

All of the HRI observations presented here show very small deviations between the optical position of the A-type star and the position of the associated X-ray source. The values range from less than $1^{\prime \prime}$ to $5^{\prime \prime}$ which is less than the spatial resolution of the HRI. All of the discussed stars are single stars or binary systems with a separation which is large enough to be resolved by the 


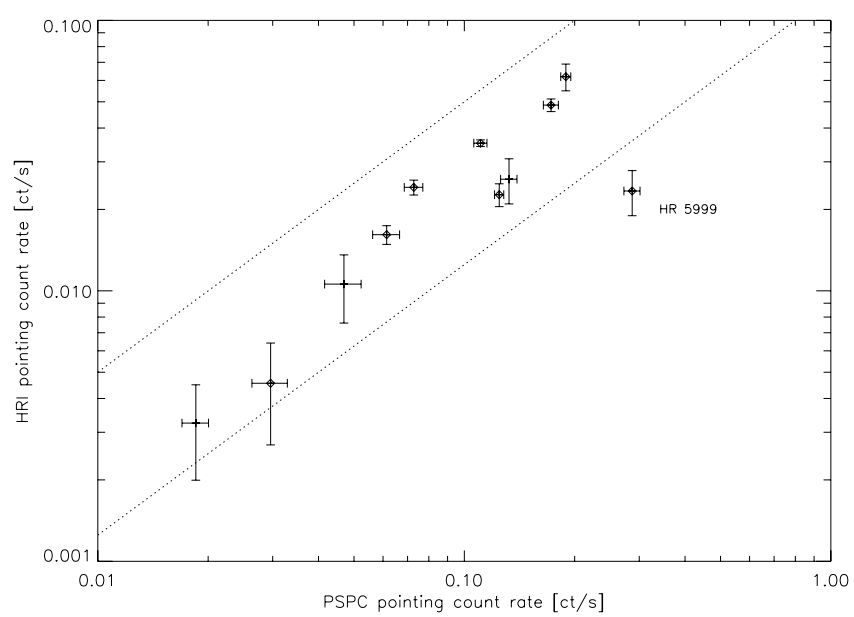

Fig. 6. Comparison of X-ray count rates for 14 stars detected both in the PSPC pointing program and the HRI pointing program. The two dotted lines indicate a factor 2 variation from unity. Single and double stars are represented by crosses and diamonds respectively. Stars with large deviations from the regression line are identified.

HRI. Objects with associated X-ray source near the edge of the detector, and therefore less accurate positions, are also excluded.

\subsubsection{HR 191}

This system is a visual binary with a separation of $19.8^{\prime \prime}$, according to the Washington Visual Double Star Catalog, with spectral types of A0IV and K2-5V. Two HRI observations of HR 191 have been carried out, one in 1991 with an exposure time of $2.1 \mathrm{ks}$ and a second in 1995 with $5.3 \mathrm{ks}$. Both observations show a weak X-ray source near the position of HR 191. While the K-star could not be detected in the first observation in 1991, a weak source is found at the position of the K-star in 1995, possibly due to a flare. The positional deviations of the X-ray sources with respect to their optical counterparts are less than one arcsecond for both stars. Unfortunately, the count rate of the A-star associated source is too low and the exposure time is too short for a significant statistical test for variability, which would be an indicator for the presence of a hidden late-type companion. The $\mathrm{X}$-ray luminosity of $1.7 \times 10^{29} \mathrm{erg} / \mathrm{s}$ is in the range that can be explained by an active late-type companion and indeed, Gerbaldi et al. (1999) mention this star as a possible astrometric binary.

\subsubsection{HR 433}

As for the previous system, HR 433 is a binary system composed of an A0V and an early K-star. The separation is $22^{\prime \prime}$ and the deviation of the position of the X-ray source from the A-type star is about $5^{\prime \prime}$. Only one source was found in the X-ray observations with an exposure time of $4.8 \mathrm{ks}$. While an X-ray luminosity of $1.1 \times 10^{29} \mathrm{erg} / \mathrm{s}$ is clearly consistent with an active late-type star, the statistical variability tests show no significant signature of variability, which would have indicated such a hidden active late-type star. The values for the radial velocity vary from -7 to $12 \mathrm{~km} \mathrm{~s}^{-1}$, and this might be a hint for a yet undetected companion.

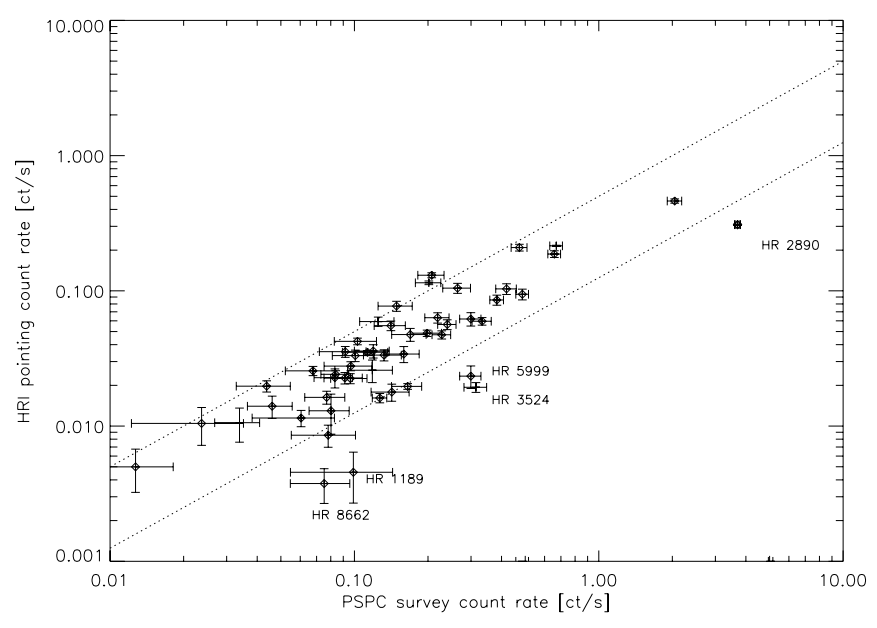

Fig. 7. Comparison of X-ray count rates for 55 stars detected both in the PSPC survey program and the HRI pointing program. The two dotted lines indicate a factor 2 variation from unity. Single and double stars are represented by crosses and diamonds respectively. Stars with large deviations from the regression line are identified.

\subsubsection{HR 778}

The Hipparcos Catalog lists this A6V star as a potential astrometric binary with a short period, but no other publication gives any information about a possible companion. The only X-ray source in the HRI image is separated from the optical position of the A-type by less than one arcsecond. During the observations, which took place in July 1997 (5.4 ks) and December 1997 and January 1998 (27.6 ks combined) the $L_{X}$ of $1.7 \times 10^{28} \mathrm{erg} / \mathrm{s}$ led to count rates which were too low for statistical tests of variability. The two measurements of the radial velocity give consistent values of $3 \mathrm{~km} \mathrm{~s}^{-1}$.

\subsubsection{HR 789}

HR 789 is again a visual binary system consisting of A2V primary accompanied by an early $M$ dwarf with a separation of $24^{\prime \prime}$. The ROSAT HRI image with an exposure time of $1.5 \mathrm{ks}$, presented in Fig. 8., clearly shows two X-ray sources at the optical positions of the stars, with the brighter X-ray source being associated with the position of the A-type star. While the Kolmogorov test showed no clear signs of variability, an additional $\chi^{2}$ test gives a probability of less than $5 \%$ that this source is constant. The $L_{\mathrm{x}}$ of $9.2 \times 10^{29} \mathrm{erg} / \mathrm{s}$ is consistent with an active late-type star. The rotational velocity of $190 \mathrm{~km} \mathrm{~s}^{-1}$ contradicts a synchronously rotating companion, but Buscombe \& Morris (1961) found variations in the radial velocity leading to the possibility of a non-synchronously rotating companion.

\subsubsection{HR 2174}

This A3V star is a member of a triple system with its closest companion at a distance of 29.2". Only one source was found near the optical position of the A-type star. During the two HRI observations in March 1995 (1.8 ks) and March 1996 (3.8 ks) this X-ray source has a deviation of one and two arcseconds to the optical position of HR 2174 and is therefore associated with the A-type star. As in the two cases mentioned above, the count rates are too low and the exposure times are too short to allow any significant statements about the variability of the X-ray 


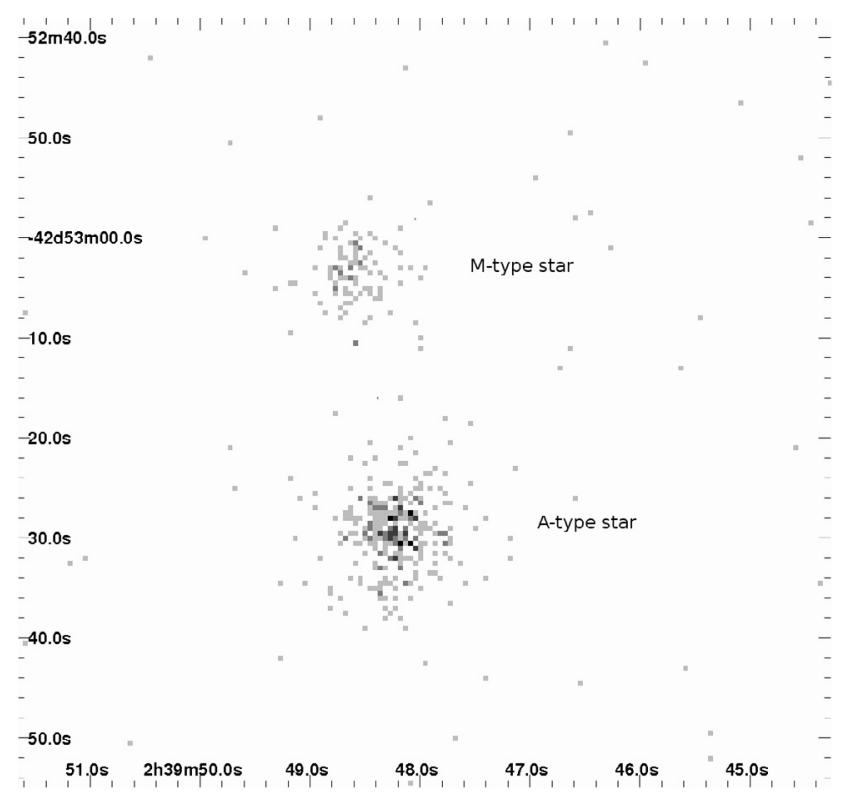

Fig. 8. ROSAT-HRI image of HR 789. The separation between the brighter X-ray source and the optical position of the A-type star is about an arcsecond.

source. With $2.3 \times 10^{30} \mathrm{erg} / \mathrm{s}$ the X-ray luminosity is at the upper limit for late-type stars. The fast rotational and constant radial velocity are indicators against an undetected companion.

\subsubsection{HR 3321}

This star is an A5III-IV star with no known companion. The HRI observations took place during April 1998 and show one X-ray source, separated by $3^{\prime \prime}$ from the optical position of HR 3321. The combined exposure time of the three observation blocks is $6.4 \mathrm{ks}$. The statistical test shows a significant variability during one of these observations. Additionally, the value of the rotational velocity is $11 \mathrm{~km} \mathrm{~s}^{-1}$ and therefore too low to be an indicator against a late-type companion. The radial velocity is constant at a value of $27.3 \mathrm{~km} \mathrm{~s}^{-1}$.

\subsubsection{HR 6681}

This A0V star is a member of a triple system with a separation of $20.8^{\prime \prime}$ from its closest companion, most likely an early G-type star. In the ROSAT HRI observations with an exposure time of $2.9 \mathrm{ks}$ carried out in September 1994, two X-ray sources with a separation of $16^{\prime \prime}$ are visible and the position of the brighter $\mathrm{X}$-ray source agrees with the optical position of the A-type star to better than $3^{\prime \prime}$. The statistical test showed no significant signs for or against variability and measurements of the radial velocity have not been carried out so far.

\subsubsection{HR 8463}

HR 8463 is an A5V star and a member of a quadruple system with a separation of 23.2" from its nearest companion. Two $\mathrm{X}$-ray sources are detected in the vicinity of the A-type star and the weaker source has a deviation of less than an arcsecond to the optical position of the A-type star. The observation is divided in four parts with a total exposure time of $6.4 \mathrm{ks}$; in three of them statistical tests do not show any signs of variability and in the forth one the tests do not give a significant result. With a distance of $55 \mathrm{pc}$ and a count rate of $0.021 \pm 0.002 \mathrm{cts} / \mathrm{s}$ the
$L_{\mathrm{X}}$ amounts to $1.8 \times 10^{29} \mathrm{erg} / \mathrm{s}$. The varying values for the radial velocity of -2 to $-16.2 \mathrm{~km} \mathrm{~s}^{-1}$ might be an indicator for a companion.

\subsection{Further comments on HRI observations}

From the six known and optically separated binary systems two companions have not been found in the observations, even though the separation of the stars is large enough to be resolved by the HRI. The reason for this is most likely the fact that at a distance of $67 \mathrm{pc}$ in the case of HR 433 and 186 pc for HR 2174 late-type stars are hardly detectable, even in a very active state.

HR 2015 is a single star separated from a X-ray source by less than $3^{\prime \prime}$. This source is listed in the HRI catalog but could not be found by our source-detection routines. We therefore flagged HR 2015 as an uncertain candidate and excluded it from the previous discussion.

\section{Summary and conclusion}

The correlation of the Bright Star Catalogue with the ROSAT X-ray catalogs results in a list of 312 bright A-type stars which can be positionally associated with an X-ray source. The overall ROSAT detection rate of bright A-type stars lies between $10-15 \%$ in the spectral range $\mathrm{A} 0-\mathrm{A} 9$, with a steep increase in detection rates among F-type stars. Those 312 A-type stars whose associated X-ray sources are listed in the ROSAT all-sky survey or the pointed observations catalog have been further investigated for signatures of the presence of late-type companions.

As a result of the evaluation of the distribution of seemingly $\mathrm{X}$-ray active stars in the different spectral subgroups it is obvious that there is neither a connection between the spectral type and the frequency of X-ray active stars nor an indication for a connection between spectral peculiarities and X-ray activity. On closer inspection, this is no real surprise. Due to the fact that a significant portion of the observed X-ray emission comes, without much doubt, from late-type companions and these companions are distributed equally on the different spectral classes, a concentration of X-ray activity on a few spectral classes would indeed be astonishing. Otherwise an increased X-ray frequency in a group of spectral peculiar stars would have given a hint about a possible mechanism for X-ray production, such as in the magnetically-confined wind shock model proposed by Babel \& Montmerle (1997). Actually none of these subgroups showed an significant increase in the X-ray detection frequency.

The examination of the count rates showed that most of the sources lack sufficient signal to obtain unambiguous results in a variability analysis. The reason for this is the fact that most of the available HRI observations are short snapshots of up to $5 \mathrm{ksec}$ to check if the target is X-ray active at all. Of the presented individual candidates, only the lightcurves of HR 789 and HR 3321 showed significant signs of variability while the lightcurve of HR 8463 was constant in three of four observation periods. We mention in passing that even clear signs of variability do not rule out the possibility that the X-ray source is the A star itself. Due to the absence of a conclusive theory for an X-ray producing mechanism at A-type stars, it is hard to predict if the A stars are variable X-ray sources or not.

For many of the X-ray detected A-type stars, information on binarity exists in partly ambiguous and partly unambiguous forms. A total of 84 of the detected X-ray stars are, however, either bona fide single stars (meaning that no information on binarity and no indications of hidden companions are available), 
or resolved multiple stars, implying that at least a third system component must exist, if the X-ray emission is not attributed to the A-type star. These 84 bona fide single stars are therefore particularly well suited candidates for follow-up observations, for example, for high resolution X-ray and IR observations to separate the suggested companion from the A-type star or radial velocity monitoring to search for companions. The list presented here may serve as a starting point for such a project.

\section{References}

Andersen, J. 1975, A\&A, 44, 445

Babel, J., \& Montmerle, T. 1997, A\&A, 323, 121

Barstow, M. A., Holberg, J. B., Fleming, T. A., et al. 1994, MNRAS, 270, 499

Berghöfer, T. W., Schmitt, J. H. M. M., \& Cassinelli, J. P. 1996, A\&AS, 118, 481

Berghöfer, T. W., Schmitt, J. H. M. M., Danner, R., \& Cassinelli, J. P. 1997, A\&A, 322, 167

Berghöfer, T. W., Schmitt, J. H. M. M., \& Hünsch, M. 1999, A\&A, 342, L17

Buscombe, W., \& Morris, P. M. 1961, MNRAS, 123, 233

Corbally, C. J. 1994, VizieR Online Data Catalog, 3117, 0

ESA 1997, VizieR Online Data Catalog, 1239, 0

Fleming, T. A., Schmitt, J. H. M. M., Barstow, M. A., \& Mittaz, J. P. D. 1991, A\&A, 246, L47

Gerbaldi, M., Faraggiana, R., Burnage, R., et al. 1999, A\&AS, 137, 273
Gudel, M., \& Schmitt, J. B. M. M. 1996, in Radio Emission from the Stars and the Sun, ed. A. R. Taylor, \& J. M. Paredes, ASP Conf. Ser., 93, 315

Güdel, M., Audard, M., Magee, H., et al. 2001, A\&A, 365, L344

Hamaguchi, K., Yamauchi, S., \& Koyama, K. 2005, ApJ, 618, 360

Hempel, M., Robrade, J., Ness, J.-U., \& Schmitt, J. H. M. M. 2005, A\&A, 440, 727

Hoffleit, D., \& Jaschek, C. 1991, The Bright star catalogue (New Haven, Conn.: Yale University Observatory), 5th rev. Ed., ed. D. Hoffleit, \& C. Jaschek,

Mamajek, E. E., Lawson, W. A., \& Feigelson, E. D. 2000, ApJ, 544, 356

Pease, D. O., Drake, J. J., \& Kashyap, V. L. 2006, ApJ, 636, 426

Randich, S., Schmitt, J. H. M. M., Prosser, C. F., \& Stauffer, J. R. 1996, A\&A, 305,785

Schmitt, J. H. M. M. 1997, A\&A, 318, 215

Schmitt, J. H. M. M. 1998, A\&A, 333, 199

Schmitt, J. H. M. M., \& Kürster, M. 1993, Science, 262, 215

Schmitt, J. H. M. M., \& Liefke, C. 2004, A\&A, 417, 651

Schmitt, J. H. M. M., Golub, L., Harnden, Jr., F. R., et al. 1985, ApJ, 290, 307

Simon, T., Drake, S. A., \& Kim, P. D. 1995, PASP, 107, 1034

Stauffer, J. R., Caillault, J.-P., Gagne, M., Prosser, C. F., \& Hartmann, L. W. 1994, ApJS, 91, 625

Stecklum, B., Eckart, A., Henning, T., \& Loewe, M. 1995, A\&A, 296, 463 Stelzer, B., Huélamo, N., Micela, G., \& Hubrig, S. 2006, A\&A, 452, 1001

Stern, R. A., Schmitt, J. H. M. M., \& Kahabka, P. T. 1995, ApJ, 448, 683

Vennes, S., Christian, D. J., \& Thorstensen, J. R. 1998, ApJ, 502, 763

Zinnecker, H., \& Preibisch, T. 1994, A\&A, 292, 152

Zombeck, M. V., Barbera, M., Collura, A., \& Murray, S. S. 1997, ApJ, 487, L69 
C. Schröder and J. H. M. M. Schmitt: X-ray emission from A-type stars, Online Material p 1

\section{Online Material}


C. Schröder and J. H. M. M. Schmitt: X-ray emission from A-type stars, Online Material p 2

Table 2. List of X-ray associated "bona fide" single or resolved multiple A-type stars.

\begin{tabular}{|c|c|c|c|c|c|c|c|c|c|c|c|c|}
\hline$\overline{\mathrm{HR}}$ & Binary & $v \sin (i)$ & Sep. & $\delta$ Mag & Components & Distance & & atalo & & $\overline{\mathrm{CR}}$ & $\pm \mathrm{CR}$ & $\overline{L_{\mathrm{x} 27}}$ \\
\hline 191 & B & & 20 & 7 & $\mathrm{~A} 0 \mathrm{IV}+\mathrm{K} 2-5 \mathrm{~V}$ & 74 & $\mathrm{R}$ & & $\mathrm{H}$ & 0.01147 & 0.00158 & 270 \\
\hline 378 & $\mathrm{~S}$ & $100-112$ & & & A3V & 67 & $\mathrm{R}$ & & & 0.01713 & 0.00787 & 78.3 \\
\hline 398 & $\mathrm{~S}$ & & & & A0Vnn & 158 & $\mathrm{R}$ & & & 0.01228 & 0.00537 & 292.1 \\
\hline 433 & B & & 22 & 5 & $\mathrm{~A} 0 \mathrm{~V}+\mathrm{K} 0 \mathrm{~V}$ & 68 & $\mathrm{R}$ & & $\mathrm{H}$ & 0.00856 & 0.00159 & 353.3 \\
\hline 710 & B & $23-31$ & 12 & 3.5 & $\mathrm{~A} 6 \mathrm{Vsp}+\mathrm{G} 5-8 \mathrm{~V}$ & 66 & $\mathrm{R}$ & & $\mathrm{H}$ & 0.03550 & 0.00327 & 422.6 \\
\hline 778 & PB & & & & A6V & 44 & & $\mathrm{P}$ & $\mathrm{H}$ & 0.01851 & 0.00154 & 21 \\
\hline 789 & B & & 24 & 10 & $\mathrm{~A} 2 \mathrm{~V}+\mathrm{M} 2-5 \mathrm{~V}$ & 40 & $\mathrm{R}$ & & $\mathrm{H}$ & 0.2090 & 0.01200 & 924 \\
\hline 817 & S & & & & A1V & 107 & $\mathrm{R}$ & & & 0.04391 & 0.01295 & 554.6 \\
\hline 943 & $\mathrm{~S}$ & $80-90$ & & & A5V & 58 & $\mathrm{R}$ & & & 0.04234 & 0.01681 & 137.9 \\
\hline 1014 & $\mathrm{~S}$ & 79 & & & A3V & 58 & & $\mathrm{P}$ & & 0.02082 & 0.00226 & 50.2 \\
\hline 1039 & $\mathrm{~S}$ & 65 & & & A0Vs & 104 & $\mathrm{R}$ & & & 0.03048 & 0.01085 & 299.9 \\
\hline 1196 & $\mathrm{~S}$ & $35-44$ & & & A5m & 60 & $\mathrm{R}$ & & & 0.04111 & 0.01120 & 141.0 \\
\hline 1314 & $\mathrm{~S}$ & $230-249$ & & & $\mathrm{~A} 2 \mathrm{Vn}$ & 108 & $\mathrm{R}$ & & & 0.03234 & 0.00957 & 498.5 \\
\hline 1700 & $\mathrm{~S}$ & & & & A1V & 130 & $\mathrm{R}$ & & & 0.01966 & 0.00737 & 358.0 \\
\hline 1706 & B & & $12.6 / 14.6$ & $6 / 3$ & $\mathrm{~A} 9 \mathrm{IV}+\mathrm{K} 2+\mathrm{F}+\mathrm{WD}$ & 82 & $\mathrm{R}$ & & $\mathrm{H}$ & 5.051 & 0.1068 & 13211.6 \\
\hline 1732 & S & $40-49$ & & & $\mathrm{~A} 0 \mathrm{pSi}$ & 137 & & $\mathrm{P}$ & & 0.02576 & 0.00168 & 343.7 \\
\hline 1940 & S & $14-15$ & & & $\mathrm{~A} 8 \mathrm{Vs}$ & 41 & $\mathrm{R}$ & & & 0.01679 & 0.00761 & 19.2 \\
\hline 2174 & B & & $29.2 / .$. & $1.2 / 3.2$ & $\mathrm{~A} 3 \mathrm{~V}+\mathrm{A} 0$ & 187 & $\mathrm{R}$ & & $\mathrm{H}$ & 0.02279 & 0.00366 & 3351 \\
\hline 2180 & S & $225-264$ & & & $\mathrm{~A} 0 \mathrm{Vn}$ & 78 & $\mathrm{R}$ & & & 0.02316 & 0.00804 & 185.8 \\
\hline 2209 & $\mathrm{~S}$ & $220-238$ & & & $\mathrm{~A} 0 \mathrm{Vn}$ & 54 & $\mathrm{R}$ & & & 0.04748 & 0.01412 & 120.4 \\
\hline 2265 & $\mathrm{~S}$ & 249 & & & A2-3V & 62 & $\mathrm{R}$ & & & 0.5004 & 0.02675 & 1858.1 \\
\hline 2350 & $\mathrm{~S}$ & & & & A5V & 69 & & $\mathrm{P}$ & & 0.00796 & 0.00059 & 27.3 \\
\hline 2351 & $S$ & $35-44$ & & & A9IV & 61 & $\mathrm{R}$ & $\mathrm{P}$ & & 0.05592 & 0.0 & 402.3 \\
\hline 2658 & S & & & & $\mathrm{A} 0 \mathrm{~V}$ & 125 & $\mathrm{R}$ & & & 0.02326 & 0.00855 & 329.1 \\
\hline 2683 & $\mathrm{~S}$ & & & & $\mathrm{~A} 0 \mathrm{pSi}$ & 87 & $\mathrm{R}$ & & & 0.02306 & 0.00863 & 186.7 \\
\hline 2709 & $\mathrm{~S}$ & 62 & & & A0III & 255 & $\mathrm{R}$ & & & 0.02186 & 0.00799 & 1023.4 \\
\hline 2720 & S & & & & $\mathrm{A} 8 \mathrm{~V}$ & 48 & $\mathrm{R}$ & & & 0.05062 & 0.01390 & 74.6 \\
\hline 2776 & $\mathrm{~S}$ & $83-94$ & & & $\mathrm{~A} 7 \mathrm{~s}$ & 34 & $\mathrm{R}$ & & & 0.03516 & 0.01172 & 24.8 \\
\hline 2869 & S & 214 & & & A1IV & 106 & $\mathrm{R}$ & & & 0.02414 & 0.01043 & 267.7 \\
\hline 3131 & $\mathrm{~S}$ & $177-245$ & & & A1V & 73 & $\mathrm{R}$ & & & 0.03230 & 0.01504 & 280.6 \\
\hline 3321 & $\mathrm{~S}$ & $11-13$ & & & A5III-IV & 53 & $\mathrm{R}$ & & $\mathrm{H}$ & 0.02777 & 0.00213 & 223.7 \\
\hline 3401 & S & $230-254$ & & & $\mathrm{~A} 2 \mathrm{Vn}$ & 110 & $\mathrm{R}$ & & & 0.01488 & 0.00712 & 156.7 \\
\hline 3649 & $\mathrm{~S}$ & $21-29$ & & & A9IVDel Del & 51 & $\mathrm{R}$ & & & 0.03393 & 0.01188 & 67.2 \\
\hline 3761 & S & 249 & & & $\mathrm{~A} 3 \mathrm{Vn}$ & 68 & $\mathrm{R}$ & & & 0.05223 & 0.01440 & 331.3 \\
\hline 4263 & S & & & & $\mathrm{A} 0 \mathrm{pSiCr}$ & 152 & $\mathrm{R}$ & & & 0.1431 & 0.02499 & 3577.0 \\
\hline 4502 & $\mathrm{~S}$ & $0-19$ & & & A0V & 64 & $\mathrm{R}$ & & & 0.09110 & 0.02831 & 270.8 \\
\hline 4554 & $\mathrm{~S}$ & $163-178$ & & & A0VSB & 26 & $\mathrm{R}$ & $\mathrm{P}$ & & 0.04295 & 0.00349 & 28.0 \\
\hline 4680 & S & $135-180$ & & & A9.5III & 55 & & $\mathrm{P}$ & & 0.01051 & 0.00094 & 23.1 \\
\hline 4886 & S & $215-233$ & & & A7V & 112 & & $P$ & & 0.01200 & 0.0 & 107.6 \\
\hline 4889 & $S$ & & & & A7III & 48 & $\mathrm{R}$ & $\mathrm{P}$ & & 0.01368 & 0.0 & 22.7 \\
\hline 4893 & B & & 22 & & A1IIIshe.+HR4892 & 93 & $\mathrm{R}$ & & $\mathrm{H}$ & 0.00499 & 0.00176 & 101.9 \\
\hline 4971 & S & & & & A9III-IV & 62 & $\mathrm{R}$ & & & 0.02244 & 0.00843 & 61 \\
\hline 5040 & $\mathrm{~S}$ & $18-26$ & & & $\mathrm{~A} 2 \mathrm{~m}$ & 64 & $\mathrm{R}$ & & & 0.1240 & 0.02372 & 398.1 \\
\hline 5062 & $\mathrm{~S}$ & $210-248$ & & & $\mathrm{~A} 5 \mathrm{~V}$ & 25 & $\mathrm{R}$ & $\mathrm{P}$ & & 0.04679 & 0.0 & 18.8 \\
\hline 5069 & $S$ & & & & $\mathrm{Ap} \mathrm{SrEuCr}$ & 88 & $\mathrm{R}$ & & & 0.03570 & 0.01525 & 137.2 \\
\hline 5107 & $\mathrm{~S}$ & $178-222$ & & & A3V & 22 & $\mathrm{R}$ & & & 0.02536 & 0.01250 & 10.7 \\
\hline 5216 & $\mathrm{~S}$ & $70-81$ & & & $\mathrm{~A} 3 \mathrm{~V}$ & 83 & $\mathrm{R}$ & & & 0.07485 & 0.01343 & 444.9 \\
\hline 5343 & $\mathrm{~S}$ & 96 & & & A8III & 49 & & $\mathrm{P}$ & & 0.00597 & 0.00092 & 10.3 \\
\hline 5357 & $\mathrm{~S}$ & 230 & & & $\mathrm{~A} 2 \mathrm{Vn}$ & 68 & $\mathrm{R}$ & & & 0.05173 & 0.01835 & 305.7 \\
\hline 5406 & $\mathrm{~S}$ & $90-102$ & & & A2IV & 72 & $\mathrm{R}$ & & & 0.07643 & 0.02048 & 387.0 \\
\hline 5468 & $S$ & $85-96$ & & & A1V & 60 & $\mathrm{R}$ & & & 0.3418 & 0.03006 & 1341.9 \\
\hline 5491 & $S$ & & & & $\mathrm{Am}$ & 64 & $\mathrm{R}$ & & & 0.04996 & 0.01835 & 148.7 \\
\hline 5511 & $S$ & $265-334$ & & & $\mathrm{~A} 0 \mathrm{~V}$ & 39 & $\mathrm{R}$ & & & 0.04499 & 0.01307 & 55.9 \\
\hline 5729 & $\mathrm{~S}$ & & & & $\mathrm{~A} 2 \mathrm{~V}$ & 67 & $\mathrm{R}$ & & & 0.2684 & 0.03963 & 1077.2 \\
\hline 5759 & $\mathrm{~S}$ & $55-65$ & & & $\mathrm{~A} 3 \mathrm{~m}$ & 92 & $\mathrm{R}$ & & & 0.00757 & 0.00353 & 45.8 \\
\hline 5845 & S & $68-78$ & & & $\mathrm{~A} 2 \mathrm{~m}$ & 53 & $\mathrm{R}$ & $\mathrm{P}$ & & 0.1003 & 0.00477 & 181.0 \\
\hline 5870 & $S$ & $120-133$ & & & A3V & 77 & $\mathrm{R}$ & & & 0.03756 & 0.01185 & 247.8 \\
\hline 6081 & $\mathrm{~S}$ & & & & A4II/III & 361 & & $\mathrm{P}$ & & 0.00849 & 0.00187 & 790.8 \\
\hline 6153 & $\mathrm{~S}$ & & & & $\mathrm{~A} 7 \mathrm{pSrEuCr}$ & 54 & & $\mathrm{P}$ & & 0.05472 & 0.00462 & 113.4 \\
\hline 6332 & $\mathrm{~S}$ & $21-29$ & & & A3IV & 90 & $\mathrm{R}$ & & & 0.00731 & 0.00380 & 86.4 \\
\hline 6386 & $\mathrm{~S}$ & $15-23$ & & & $\mathrm{~A} 0 \mathrm{~V}$ & 114 & $\mathrm{R}$ & & & 0.03536 & 0.01261 & 740.6 \\
\hline 6537 & $S$ & & & & $\mathrm{~A} 0 \mathrm{~V}$ & 118 & $\mathrm{R}$ & & & 0.04082 & 0.0189 & 461.9 \\
\hline 6539 & S & & & & $\mathrm{A} 0 \mathrm{~V}$ & 104 & $\mathrm{R}$ & $\mathrm{P}$ & & 0.07882 & 0.0 & 915.0 \\
\hline 6681 & B & & $20.8 / 33.7$ & $2.2 / 6.5$ & $\mathrm{~A} 0 \mathrm{~V}+\mathrm{G} 0-2 \mathrm{~V}$ & 76 & $\mathrm{R}$ & & $\mathrm{H}$ & 0.01784 & 0.00258 & 950.9 \\
\hline 6782 & B & & 14.2 & & $\mathrm{~A} 3 \mathrm{~V}$ & 51 & $\mathrm{R}$ & & $\mathrm{H}$ & 0.4866 & 0.0 & 81 \\
\hline 7012 & $\mathrm{~S}$ & & & & A5IV-V & 29 & $\mathrm{R}$ & & & 0.09705 & 0.03182 & 70.8 \\
\hline 7018 & $S$ & 30 & & & $\mathrm{~A} 0 \mathrm{~V}$ & 131 & $\mathrm{R}$ & & & 0.01584 & 0.00307 & 303.6 \\
\hline 7085 & S & $90-102$ & & & A1V & 176 & & $\mathrm{P}$ & & 0.00967 & 0.00291 & 216.6 \\
\hline 7250 & $\mathrm{~S}$ & $35-44$ & & & A4III & 102 & $\mathrm{R}$ & & & 0.01807 & 0.00725 & 134.8 \\
\hline 7312 & $\mathrm{~S}$ & $55-64$ & & & A9V & 27 & $\mathrm{R}$ & & & 0.1271 & 0.00981 & 86.9 \\
\hline 7384 & $S$ & $170-186$ & & & $\mathrm{~A} 0 \mathrm{~V}$ & 123 & $\mathrm{R}$ & & & 0.07768 & 0.01714 & 1210.4 \\
\hline 7416 & $S$ & & & & $\mathrm{~A} 0 \mathrm{p}(\mathrm{CrEuSr})$ & 134 & $\mathrm{R}$ & & & 0.03580 & 0.01333 & 532.1 \\
\hline 7423 & S & $165-180$ & & & A3V & 95 & $\mathrm{R}$ & & & 0.01309 & 0.00464 & 189.8 \\
\hline 7498 & S & & & & A4III & 42 & $\mathrm{R}$ & & & 0.08967 & 0.03027 & 111 \\
\hline 7634 & $S$ & 175 & & & $\mathrm{~A} 4 \mathrm{Vn}$ & 86 & $\mathrm{R}$ & & & 0.01048 & 0.00380 & 70.1 \\
\hline 7702 & $\mathrm{~S}$ & 190-207 & & & A3V & 200 & $\mathrm{R}$ & & & 0.01118 & 0.00461 & 607.6 \\
\hline 7730 & $S$ & & 337 & & A5IIIn & 220 & $\mathrm{R}$ & & & 0.02710 & 0.00671 & 939.9 \\
\hline 7734 & $S$ & $220-238$ & & & $\mathrm{~A} 0 \mathrm{~V}$ & 310 & & $\mathrm{P}$ & & 0.00248 & 0.00068 & 170.7 \\
\hline 7826 & S & & & & $\mathrm{A} 3 \mathrm{~V}$ & 83 & $\mathrm{R}$ & & & 0.02057 & 0.00765 & 56.9 \\
\hline 8463 & B & & 23.21 & $5 /$ & A5V & 55 & $\mathrm{R}$ & & $\mathrm{H}$ & 0.02248 & 0.00192 & 219.6 \\
\hline 8578 & S & $45-81$ & & & $\mathrm{~A} 2 \mathrm{~m}$ & 63 & $\mathrm{R}$ & & & 0.02248 & 0.00584 & 81.1 \\
\hline 8675 & $S$ & & & & $\mathrm{~A} 3 \mathrm{~V}$ & 40 & & $\mathrm{P}$ & & 0.01243 & 0.00206 & 13.4 \\
\hline 9060 & S & & & & A1IV & 63 & $\mathrm{R}$ & & & 0.07669 & 0.01949 & 314.3 \\
\hline
\end{tabular}


C. Schröder and J. H. M. M. Schmitt: X-ray emission from A-type stars, Online Material p 3

\begin{tabular}{|c|c|c|c|c|c|c|c|c|c|c|}
\hline HR & binary & $v \sin (i)$ & sep. & $\delta \mathrm{mag}$ & Components & Distance & Catalog & $\mathrm{CR}$ & $\pm \mathrm{CR}$ & $L_{\mathrm{x} 27}$ \\
\hline 9062 & $\mathrm{~S}$ & $187-191$ & & & $\mathrm{~A} 1 \mathrm{~V}$ & 49 & & 0.05986 & 0.01889 & 102.2 \\
\hline
\end{tabular}


C. Schröder and J. H. M. M. Schmitt: X-ray emission from A-type stars, Online Material p 4

Table 3. List of X-ray associated A-type stars in binary or multiple systems or with hints of hidden companions.

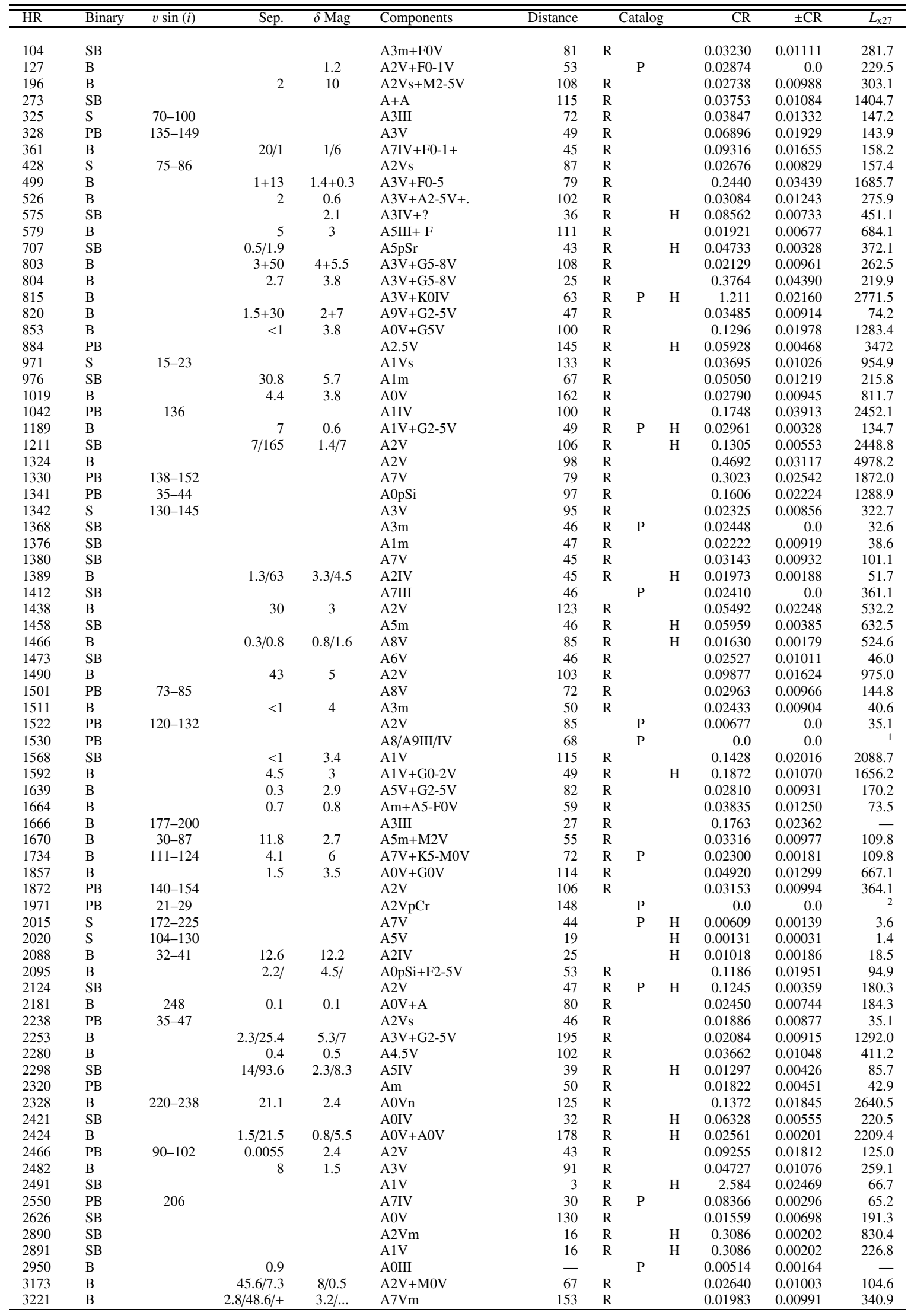

${ }^{1}$ Simon et al. (1995) calculate an $L_{\mathrm{x}}$ of $33 \times 10^{27} \mathrm{erg} / \mathrm{s}$ from PSPC pointing observations. The Second ROSAT Source Catalog of Pointed Observation (2001) lists false detections in the field and therefor no count rate for the source.

2 In The Second ROSAT Source Catalog of Pointed Observation (2001) this source is flagged as suspect in lightcurve, variability and spectrum. No count rate for the source is listed. 
C. Schröder and J. H. M. M. Schmitt: X-ray emission from A-type stars, Online Material p 5

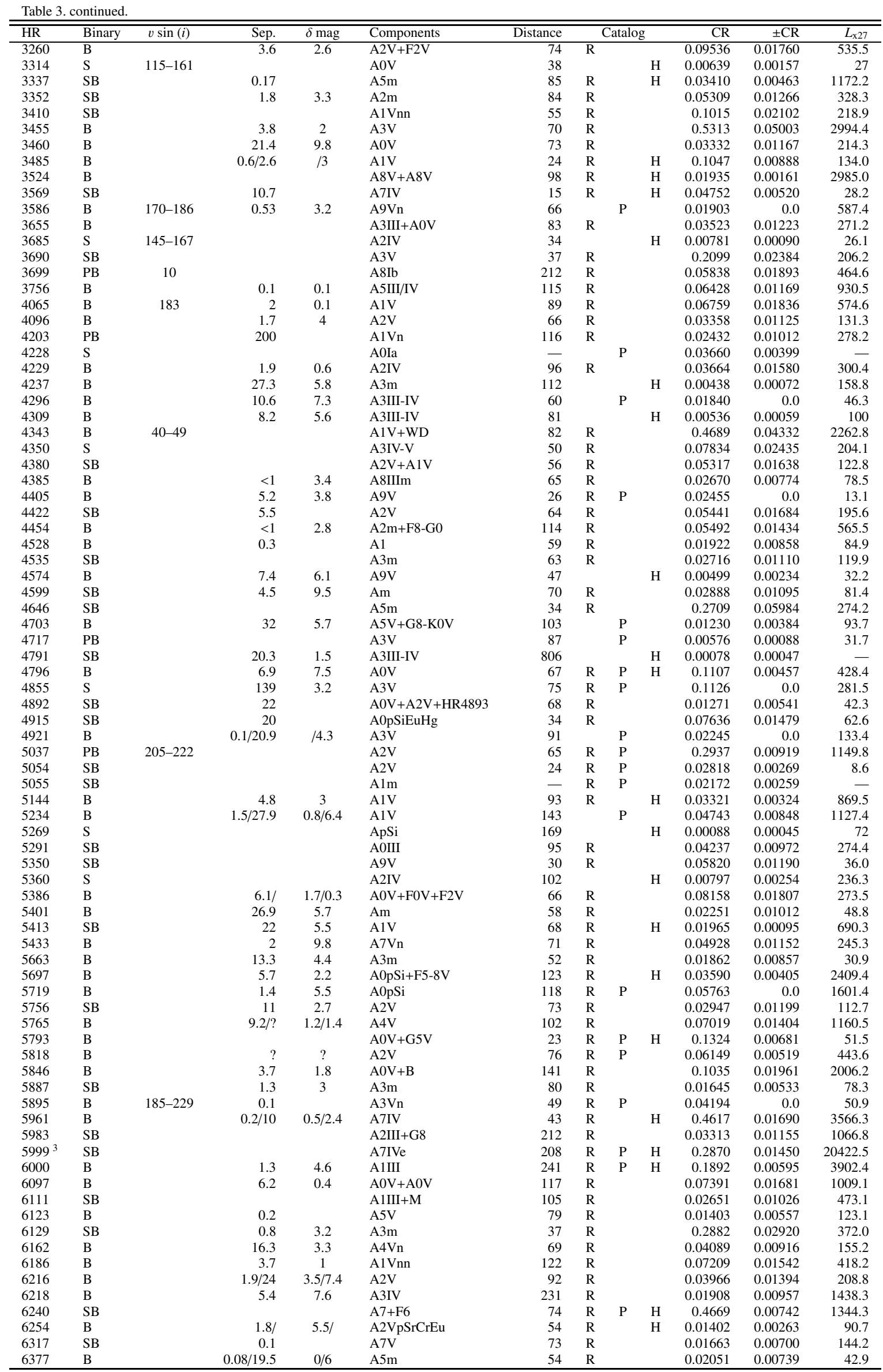

${ }^{3}$ See Zinnecker \& Preibisch (1994) and Hamaguchi et al. (2005). 
C. Schröder and J. H. M. M. Schmitt: X-ray emission from A-type stars, Online Material p 6

\begin{tabular}{|c|c|c|c|c|c|c|c|c|c|c|c|c|}
\hline $\begin{array}{ll}\mathrm{HR} \\
6435\end{array}$ & $\begin{array}{l}\text { Binary } \\
\mathrm{B}\end{array}$ & $\begin{array}{l}v \sin (i) \\
210-228\end{array}$ & $\begin{array}{r}\text { Sep. } \\
1.6 / 11.5\end{array}$ & $\begin{array}{c}\delta \text { mag } \\
1 / 4.9\end{array}$ & $\begin{array}{l}\text { Components } \\
\mathrm{A} 0 \mathrm{~V}(\mathrm{nn})\end{array}$ & $\begin{array}{r}\text { Distance } \\
114\end{array}$ & \multicolumn{3}{|c|}{ Catalog } & $\begin{array}{r}\text { CR } \\
0.04231\end{array}$ & & $L_{\mathrm{X} 27}$ \\
\hline 6436 & SB & & 0.9 & & $\mathrm{~A} 2 \mathrm{~V}$ & 55 & $\overline{\mathrm{R}}$ & $\overline{\mathrm{P}}$ & & 0.03320 & 0.0 & 92.5 \\
\hline 6555 & SB & & & & $\mathrm{A} 4 \mathrm{~m}$ & 31 & $\mathrm{R}$ & & & 0.01433 & 0.00427 & 9.9 \\
\hline 6556 & PB & & & & A5III & 14 & $\mathrm{R}$ & & & 0.1241 & 0.01652 & 18.3 \\
\hline 6618 & PB & $130-170$ & & & $\mathrm{~A} 2 \mathrm{~V}$ & 127 & $\mathrm{R}$ & & & 0.00979 & 0.00324 & 207.7 \\
\hline 6641 & SB & & 0.1 & & $\mathrm{~A} 2 \mathrm{Vs}$ & 132 & $\mathrm{R}$ & & & 0.08195 & 0.00874 & 1661.5 \\
\hline 6771 & SB & & $25.3 / 51.7$ & $10.3 / 7.3$ & A4IVs & 25 & $\mathrm{R}$ & & & 0.02134 & 0.00982 & 8.6 \\
\hline 6781 & B & & $0.1 / 14.2$ & & A3V & 70 & $\mathrm{R}$ & & $\mathrm{H}$ & 0.09434 & 0.00861 & 2239.4 \\
\hline 6825 & PB & $25-33$ & & & $\mathrm{ApSi}$ & 1205 & $\mathrm{R}$ & & & 0.05331 & 0.01705 & - \\
\hline 6843 & B & & 0.7 & 3.5 & $\mathrm{~A} 8 \mathrm{~V}$ & 132 & $\mathrm{R}$ & & & 0.1390 & 0.02396 & 3939.3 \\
\hline 6876 & SB & & & & $\mathrm{A} 5 \mathrm{~m}$ & 58 & $\mathrm{R}$ & & & 0.04933 & 0.01029 & 145.0 \\
\hline 6923 & SB & & & & $\mathrm{A} 1 \mathrm{~V}$ & 58 & $\mathrm{R}$ & $\mathrm{P}$ & $\mathrm{H}$ & 0.06139 & 0.00518 & 373.0 \\
\hline 7124 & SB & & & & $\mathrm{A} 1 \mathrm{Vn}$ & 93 & $\mathrm{R}$ & & & 0.02501 & 0.00495 & 203.5 \\
\hline 7160 & $\mathrm{~S}$ & & & & A8V & 88 & $\mathrm{R}$ & $\mathrm{P}$ & $\mathrm{H}$ & 0.04693 & 0.00536 & 243.4 \\
\hline 7214 & PB & & & & $\mathrm{A} 4 \mathrm{~V}$ & 55 & $\mathrm{R}$ & & & 0.07833 & 0.01560 & 255.2 \\
\hline 7215 & B & & 41.9 & 5.6 & A7V & 39 & $\mathrm{R}$ & & & 0.07750 & 0.01322 & 105.3 \\
\hline 7235 & SB & & & & $\mathrm{A} 0 \mathrm{Vn}$ & 26 & $\mathrm{R}$ & & $\mathrm{H}$ & 0.05526 & 0.00451 & 84.9 \\
\hline 7313 & PB & $195-212$ & & & $\mathrm{~A} 1 \mathrm{Vn}$ & 84 & $\mathrm{R}$ & & & 0.04254 & 0.01231 & 255.3 \\
\hline 7392 & SB & & & & $\mathrm{Am}$ & 57 & $\mathrm{R}$ & & & 0.03887 & 0.01431 & 122.3 \\
\hline 7431 & SB & & & & A1mA2-F0 & 89 & $\mathrm{R}$ & & $\mathrm{H}$ & 0.01046 & 0.00326 & 134.9 \\
\hline 7557 & S & & & & A7V & 5 & $\mathrm{R}$ & $\mathrm{P}$ & & 0.1785 & 0.00357 & 6.4 \\
\hline 7562 & B & & 0.4 & 3.3 & $\mathrm{~A} 1 \mathrm{~m}$ & 117 & $\mathrm{R}$ & $P$ & & 0.0264 & 0.0 & 370.2 \\
\hline 7571 & B & & 0.15 & & $\mathrm{~A} 1 \mathrm{~V}+\mathrm{F} 8 \mathrm{IV}$ & 117 & $\mathrm{R}$ & & & 0.3836 & 0.04335 & 6865.9 \\
\hline 7610 & SB & & & & A1IV & 63 & $\mathrm{R}$ & $\mathrm{P}$ & & 0.03413 & 0.0 & 122.1 \\
\hline 7695 & B & & $4.9 / 150$ & $3-4 / 5$ & A2II-III+ & 78 & $\mathrm{R}$ & & & 0.02284 & 0.00550 & 101 \\
\hline 8140 & B & & 3 & 2.5 & $\mathrm{~A} 5 \mathrm{~V}+\mathrm{G} 0 \mathrm{~V}$ & 30 & $\mathrm{R}$ & & $\mathrm{H}$ & 0.1034 & 0.00936 & 341.3 \\
\hline 8151 & B & & 0.15 & 2.5 & A2pCrEuSr+ & 57 & $\mathrm{R}$ & & & 0.1062 & 0.01907 & 411.2 \\
\hline 8162 & B & & 209.2 & 8 & A7IV & 15 & $\mathrm{R}$ & $\mathrm{P}$ & & 0.02026 & 0.00278 & 7.1 \\
\hline 8210 & SB & & & & $\mathrm{A} 8 \mathrm{Vm}+\mathrm{WD}$ & 46 & $\mathrm{R}$ & & & 1.052 & 0.06780 & 3631.1 \\
\hline 8263 & B & & 31.3 & 3.3 & $\mathrm{~A} 2 \mathrm{~V}$ & 122 & $\mathrm{R}$ & & & 0.02600 & 0.01072 & 322.3 \\
\hline 8266 & PB & $185-201$ & & & A5V & 63 & $\mathrm{R}$ & & & 0.01405 & 0.00626 & 36.6 \\
\hline 8307 & B & & $1.4 /$ & $5.4 /$ & $\mathrm{A} 0 \mathrm{~V}$ & 84 & & $\mathrm{P}$ & & 0.05806 & 0.00468 & 4326.6 \\
\hline 8322 & B & & & & $\mathrm{Am}$ & 12 & $\mathrm{R}$ & & & 1.655 & 0.12550 & 187.5 \\
\hline 8396 & SB & & & & $\mathrm{A} 2 \mathrm{~V}+\mathrm{K} 0 \mathrm{III}$ & 145 & $\mathrm{R}$ & & $\mathrm{H}$ & 0.03348 & 0.00316 & 505.2 \\
\hline 8417 & SB & & & & $\mathrm{A} 3 \mathrm{~m}$ & 31 & $\mathrm{R}$ & & $\mathrm{H}$ & 0.05639 & 0.00470 & 169.7 \\
\hline 8431 & PB & & & & $\mathrm{A} 2 \mathrm{~V}$ & 40 & $\mathrm{R}$ & & & 0.03442 & 0.01345 & 89.6 \\
\hline 8518 & SB & & & & $\mathrm{A} 0 \mathrm{~V}$ & 48 & $\mathrm{R}$ & & & 0.05508 & 0.01707 & 92.9 \\
\hline 8533 & B & & $0.5 /$ & $0.2 /$ & $\mathrm{A} 0 \mathrm{~V}$ & 130 & $\mathrm{R}$ & $\mathrm{P}$ & & 0.01041 & 0.0 & 448.2 \\
\hline 8576 & B & & 30.3 & 2 & $\mathrm{~A} 0 \mathrm{~V}$ & 45 & $\mathrm{R}$ & & & 0.06447 & 0.01814 & 88.5 \\
\hline 8598 & B & & $9.5 /$ & $3 /$ & $\mathrm{A} 0 \mathrm{~V}$ & 123 & $\mathrm{R}$ & & & 0.01753 & 0.00758 & 104.9 \\
\hline 8600 & PB & & & & $\mathrm{A} 3 \mathrm{Vn}$ & 69 & $\mathrm{R}$ & & & 0.03796 & 0.01648 & 169.9 \\
\hline 8662 & SB & & & & A9IIIm & 74 & $\mathrm{R}$ & & $\mathrm{H}$ & 0.00376 & 0.00108 & 14.8 \\
\hline 8724 & B & & 3.9 & 2.9 & $\mathrm{~A} 3 \mathrm{Vs}$ & 82 & $\mathrm{R}$ & & & 0.04692 & 0.01266 & 337.9 \\
\hline 8738 & $\mathrm{~S}$ & & & & A1V & 96 & & $\mathrm{P}$ & & 0.01433 & 0.00 & 90.2 \\
\hline 8799 & S & & & & A5V & 40 & $\mathrm{R}$ & & & 0.02123 & 0.00783 & 14.7 \\
\hline 8865 & B & & 1.2 & 4 & $\mathrm{~A} 0 \mathrm{~V}$ & 76 & $\mathrm{R}$ & & $\mathrm{H}$ & 0.07713 & 0.00658 & 834.0 \\
\hline 8884 & B & & 13.2 & 3.5 & $\mathrm{~A} 5 \mathrm{Vn}$ & 80 & $\mathrm{R}$ & & & 0.01986 & 0.00827 & 121.2 \\
\hline 8963 & B & & 27.7 & 6.2 & $\mathrm{~A} 1 \mathrm{Vn}$ & 73 & & & $\mathrm{H}$ & 0.00203 & 0.00086 & 31.3 \\
\hline 9016 & B & & $3.3 /$ & $7 /$ & $\mathrm{A} 0 \mathrm{~V}$ & 44 & $\mathrm{R}$ & $\mathrm{P}$ & $\mathrm{H}$ & 0.1724 & 0.00813 & 379.4 \\
\hline 9039 & PB & $156-180$ & & & $\mathrm{~A} 4 \mathrm{Vn}$ & 58 & $\mathrm{R}$ & & & 0.03021 & 0.01058 & 99.6 \\
\hline
\end{tabular}

